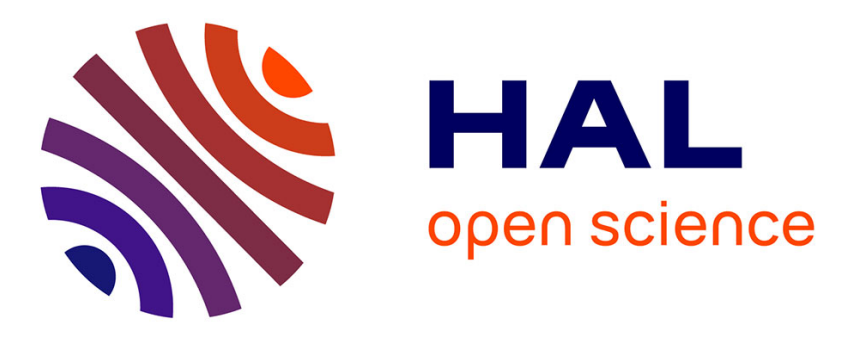

\title{
Singularity Analysis of the 4-RUU Parallel Manipulator using Grassmann-Cayley Algebra
}

\author{
Semaan Amine, Mehdi Tale Masouleh, Stéphane Caro, Philippe Wenger, \\ Clément M Gosselin
}

\section{- To cite this version:}

Semaan Amine, Mehdi Tale Masouleh, Stéphane Caro, Philippe Wenger, Clément M Gosselin. Singularity Analysis of the 4-RUU Parallel Manipulator using Grassmann-Cayley Algebra. Transactions of the Canadian Society for Mechanical Engineering, 2011, 35 (5), pp.515-528. 10.1139/tcsme-20110031 . hal-00684999

\section{HAL Id: hal-00684999 \\ https://hal.science/hal-00684999}

Submitted on 3 Apr 2012

HAL is a multi-disciplinary open access archive for the deposit and dissemination of scientific research documents, whether they are published or not. The documents may come from teaching and research institutions in France or abroad, or from public or private research centers.
L'archive ouverte pluridisciplinaire HAL, est destinée au dépôt et à la diffusion de documents scientifiques de niveau recherche, publiés ou non, émanant des établissements d'enseignement et de recherche français ou étrangers, des laboratoires publics ou privés. 


\title{
SINGULARITY ANALYSIS OF THE 4-RUUU PARALLEL MANIPULATOR USING GRASSMANN-CAYLEY ALGEBRA
}

\author{
Semaan Amine ${ }^{1}$, Mehdi Tale Masouleh ${ }^{2}$, Stéphane Caro ${ }^{1}$, Philippe Wenger ${ }^{1}$, Clément Gosselin ${ }^{2}$ \\ ${ }^{1}$ Institut de Recherche en Communications et Cybernétique de Nantes, Nantes, France \\ ${ }^{2}$ Département de Génie Mécanique, Université Laval, Québec, Canada \\ E-mail:semaan.amine@irccyn.ec-nantes.fr,mehdi.tale-masouleh.1@ulaval.ca,stephane.caro@irccyn.ec-nantes.fr, \\ philippe.wenger@irccyn.ec-nantes.fr,gosselin@gmc.ulaval.ca
}

\begin{abstract}
This paper deals with the singularity analysis of four degrees of freedom parallel manipulators with identical limb structures performing Schönflies motions, namely, three independent translations and one rotation about an axis of fixed direction. The $6 \times 6$ Jacobian matrix of such manipulators contains two lines at infinity among its six Plücker vectors. Some points at infinity are thus introduced to formulate the superbracket of Grassmann-Cayley algebra, which corresponds to the determinant of the Jacobian matrix. By exploring this superbracket, all the singularity conditions of such manipulators can be enumerated. The study is illustrated through the singularity analysis of the 4-RUUU parallel manipulator.
\end{abstract}

Keywords: parallel manipulator; singularity; Schönflies motions; Grassmann-Cayley algebra; superbracket.

\section{RÉSUMÉ}

Cet article traite de l'analyse des singularités de manipulateurs parallèles à quatre degrés de liberté ayant des jambes identiques, générant des mouvements dits de Schönflies, c.à.d. trois translations indépendantes et une rotation autour d'un axe de direction fixe. La matrice jacobienne $6 \times 6$ de ces manipulateurs contient deux lignes à l'infini parmi ses six vecteurs de Plücker. Quelques points à l'infini sont ainsi introduits pour formuler le superbracket de l'algèbre de Grassmann-Cayley qui correspond au déterminant de la matrice jacobienne. En examinant ce superbracket, toutes les conditions de singularités de ces manipulateurs peuvent être énumérées. Les contributions de l'article sont illustrées à travers l'analyse de singularités du manipulateur parallèle 4-ㅌUU.

Mots-clés: manipulateur parallèle ; singularités ; mouvements de Schönflies ; algèbre de GrassmannCayley; superbracket. 


\section{INTRODUCTION}

The singular configurations of Parallel Manipulators (PMs) are critical poses characterized by either the loss of some degrees of freedom $(d o f)$, the gain of some extra dof or the loss of stiffness. The determination of singular configurations is thus a central issue in robotics due to their major effect on the robot performance [1,2]. Lower-mobility PMs are suitable for a wide range of applications that require fewer than six dof. The classification of singularities for lower-mobility PMs has stimulated the interest of many researchers [3-6]. In this paper, the classification proposed in [4], which is similar the one proposed in [7], is adopted. Accordingly, a lower-mobility PM can exhibit three different types of singularities: (i) limb singularities, (ii) platform singularities [4], also known as constraint singularities [8] and (iii) actuation singularities, also called architecture singularities [7]. Constraint and actuation singularities are referred to as parallel singularities and are related to the rank deficiency of the $6 \times 6$ Jacobian matrix $\mathbf{J}$ of the PM that transforms the velocities of the actuators to the linear and angular velocities of the end-effector.

The determination of the parallel singularities of a PM consists in finding either the poses, yielding the singularity locus, or the conditions, yielding the configurations, for which $\mathbf{J}$ becomes rankdeficient. Generally, $\mathbf{J}$ expresses a system of screws or more precisely Plücker vectors ${ }^{3}$. In this paper, $\mathbf{J}$ is determined by using the theory of reciprocal screws [9-12]. For most manipulators, the determinant of such a matrix is highly nonlinear and unwieldy to assess even with a computer algebra system. Hence, linear algebra fails to provide satisfactory results, and therefore, the use of Grassmann Geometry (GG) [1, 13-15] or Grassmann-Cayley Algebra (GCA) [2, 16-18] can be regarded as a promising solution. The GG is a geometric approach that provides a classification for the conditions under which a set of $n$ Plücker lines spans a variety of dimension lower than $n$. On the other hand, the GCA is a systematic approach to obtain a bracket representation of the determinant of $\mathbf{J}$, called superbracket. By exploring this superbracket, it is possible to obtain a vector form and a geometrical interpretation of the parallel singularities.

Ben-Horin et al. $[2,16]$ analyzed the singularities of 6-dof PMs whose actuated joints transmit six pure actuation forces, i.e., six finite lines, to the moving platform, by means of GCA. The singularity conditions are derived from a simplified expression of the superbracket, which is obtained by using the intersection points between actuation lines. Nevertheless, their method does not consider the intersection at infinity of two parallel finite lines. Moreover, it does not apply when some Plücker vector(s) of $\mathbf{J}$ correspond to the Plücker coordinate vector(s) of a line at infinity. Recently, Kanaan et al. [17] and Amine et al. [18] filled this gap by using some properties of projective geometry in order to formulate a superbracket with points and lines at infinity, and therefore, to extend the application of GCA to lower-mobility PMs. This paper focuses on the application of GCA to provide a compact vector expression for the singularity locus of 3T1R PMs with identical limb structures.

Schönflies Motion Generators (SMGs) [19] are manipulators performing three independent translations and one rotation about an axis of fixed direction. This type of motion is required in a wide range of industrial pick and place operations such as the assembly of computer circuit boards. The

\footnotetext{
${ }^{3}$ A Plücker vector, also known as Plücker line, denotes the Plücker coordinate vector of a projective line, namely, a line in the 3-dimensional projective space.
} 
type synthesis of parallel SMGs with identical limb structures, performed in [12], leads to four kine-

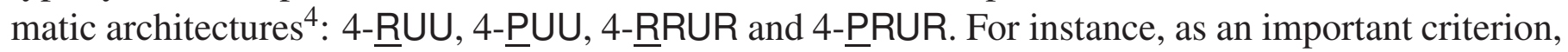
the kinematic arrangements $\underline{R} U U$ and $\underline{P} U U$ require two links whereas the other ones require at least three links. In this paper, we focus on the singularity analysis of the 4-ㅌUU PM based on GCA. The remainder of the paper is organized as follows. First, the superbracket decomposition of GCA and some fundamental concepts of the projective space $\mathbb{P}_{3}$ are recalled. Then, the 4- $\underline{R} U U$ PM is presented and its constraint analysis is performed in order to determine its Jacobian matrix $\mathbf{J}$. A superbracket of the PM is then formulated. Finally, the singularity conditions of the 4-RUU PM are enumerated and some singular configurations are illustrated.

\section{GRASSMANN-CAYLEY ALGEBRA}

The GCA was developed by H. Grassmann (1809-1877) as a calculus for linear varieties operating on extensors with the join " $\vee$ " and meet " $\wedge$ " operators. The latter are associated with the span and intersection of vector spaces of extensors characterized by their step. GCA makes it possible to work at the symbolic level, and therefore, to produce coordinate-free algebraic expressions for the singularity conditions of spatial PMs. For further details on GCA, the reader is referred to $[2,16,20]$ and references therein.

\subsection{Extensors}

Let $V$ be a $n$-dimensional vector space over the field of $\mathbb{R}, U$ be a $k$-dimensional subspace of $V$ and $\left\{\mathbf{u}_{1}, \mathbf{u}_{2}, \ldots, \mathbf{u}_{k}\right\}$ be a basis of $U$. Let $\mathbf{P}$ be the Plücker coordinate vector of $U$, regarded at the symbolic level, namely, as a vector of the $\left(\begin{array}{l}n \\ k\end{array}\right)$-dimensional vector space $V^{(k)}$. In this case, $\mathbf{P}$ is called k-extensor or decomposable k-tensor and is denoted by:

$$
\mathbf{P}=\vee\left(\mathbf{u}_{1}, \mathbf{u}_{2}, \ldots, \mathbf{u}_{k}\right)=\mathbf{u}_{1} \vee \mathbf{u}_{2} \vee \ldots \vee \mathbf{u}_{k} .
$$

The vector subspace $U$, also denoted by $\overline{\mathbf{P}}$, is called the support of $\mathbf{P}$, and the scalar $k$ is called the step of the extensor. In the 4-dimensional vector space $V$ associated with the 3-dimensional projective space $\mathbb{P}^{3}$, extensors of step 1,2 and 3 correspond to points, lines and planes, respectively.

\subsection{Join operator}

Let $\mathbf{A}=a_{1} \vee a_{2} \vee \ldots a_{k}$ et $\mathbf{B}=b_{1} \vee b_{2} \vee \ldots b_{j}$ (or simply $\mathbf{A}=a_{1} a_{2} \ldots a_{k}$ and $\mathbf{B}=b_{1} b_{2} \ldots b_{j}$ ), be two extensors in $V$ of steps $j$ and $k$, respectively, with $j+k \leq n$. The join of $\mathbf{A}$ and $\mathbf{B}$, is the $(j+k)-$ extensor $\mathbf{A} \vee \mathbf{B}$ given by:

$$
\mathbf{A} \vee \mathbf{B}=\mathrm{a}_{1} \mathrm{a}_{2} \ldots \mathrm{a}_{\mathrm{k}} \mathrm{b}_{1} \mathrm{~b}_{2} \ldots \mathrm{b}_{\mathrm{j}}
$$

If vectors $\mathrm{a}_{1}, \mathrm{a}_{2}, \ldots, \mathrm{a}_{k}, \mathrm{~b}_{1}, \mathrm{~b}_{2}, \ldots, \mathrm{b}_{j-1}$ and $\mathrm{b}_{j}$ are linearly dependent, then $\mathbf{A} \vee \mathbf{B}=0$. If they are independent, then $\overline{\mathbf{A} \vee \mathbf{B}}=\overline{\mathbf{A}}+\overline{\mathbf{B}}=\operatorname{span}(\overline{\mathbf{A}} \cup \overline{\mathbf{B}})$, the space vector spanned by $\overline{\mathbf{A}} \cup \overline{\mathbf{B}}$.

\footnotetext{
${ }^{4} \mathrm{R}, \mathrm{P}$ and $\mathrm{U}$ stand for a revolute joint, a prismatic joint and a universal joint, respectively, while an underline is used to denote the actuated joints.
} 
The join of two extensors corresponds to the sum of their associated vector spaces. The join operation is anti-commutative:

$$
\mathbf{A} \vee \mathbf{B}=(-1)^{k j} \mathbf{B} \vee \mathbf{A}
$$

The vector space $V^{(k)}$ is closed under the addition but not closed under the join operation. If we combine the vector spaces $V^{(k)},(k=1,2, \ldots, n)$, used to represent the vector subspaces of $V$, into another vector space defined as:

$$
\Lambda(V)=V^{(0)} \oplus V^{(1)} \oplus \ldots \oplus V^{(n)}
$$

then $\Lambda(V)$ will be closed under both the addition and the join operations. The vector space $\Lambda(V)$ with the join operation is known as the exterior algebra on $V$.

\subsection{Meet operator}

Let $\mathbf{A}=\mathrm{a}_{1} \vee \mathrm{a}_{2} \vee \ldots \mathrm{a}_{\mathrm{k}}=\mathrm{a}_{1} \mathrm{a}_{2} \ldots \mathrm{a}_{\mathrm{k}}$ and $\mathbf{B}=\mathrm{b}_{1} \mathrm{~b}_{2} \ldots \mathrm{b}_{\mathrm{j}}$ be two extensors with $j+k \geq n$.

The meet of $\mathbf{A}$ and $\mathbf{B}$ is defined as:

$$
\begin{aligned}
\mathbf{A} \wedge \mathbf{B} & =\sum_{\sigma} \operatorname{sign}(\sigma)\left[\mathrm{a}_{\sigma(1)} \mathrm{a}_{\sigma(2)} \ldots \mathrm{a}_{\sigma(\mathrm{n}-\mathrm{j})} \mathrm{b}_{1} \ldots \mathrm{b}_{\mathrm{j}}\right] \mathrm{a}_{\sigma(\mathrm{n}-\mathrm{j}+1)} \mathrm{a}_{\sigma(\mathrm{n}-\mathrm{j}+2)} \ldots \mathrm{a}_{\sigma(\mathrm{k})} \\
& =\sum_{\sigma} \operatorname{sign}(\sigma)\left[\dot{\mathrm{a}}_{1} \dot{\mathrm{a}}_{2} \ldots \dot{\mathrm{a}}_{\mathrm{n}-\mathrm{j}} \mathrm{b}_{1} \mathrm{~b}_{2} \ldots \mathrm{b}_{\mathrm{h}}\right] \dot{\mathrm{a}}_{\mathrm{n}-\mathrm{j}+1} \ldots \dot{\mathrm{a}}_{\mathrm{k}}
\end{aligned}
$$

The brackets stand for determinants, and the sum is taken over all permutations $\sigma$ of $\{1,2, \ldots, k\}$ such that: $\sigma(1)<\sigma(2)<\ldots<\sigma(n-j)$ and $\sigma(n-j+1)<\sigma(n-j+2)<\ldots<\sigma(k)$. An alternative notation is to use a dot over the permuted elements [20] instead of using $\sigma$. Equation (5) is called shuffle formula, and is very useful for practical applications of GCA. If $\mathbf{A} \neq 0, \mathbf{B} \neq 0$ and $\overline{\mathbf{A}} \cup \overline{\mathbf{B}}$ spans $V$, then $\overline{\mathbf{A} \wedge \mathbf{B}}=\overline{\mathbf{A}} \cap \overline{\mathbf{B}}$. In this case, $\mathbf{A} \wedge \mathbf{B}$ is an extensor of step $k+j-n$.

The meet of two extensors is always an extensor. The meet of two extensors corresponds to the intersection of their associated vector spaces. The meet operation is anti-commutative:

$$
\mathbf{A} \wedge \mathbf{B}=(-1)^{(n-k)(n-j)} \mathbf{B} \wedge \mathbf{A}
$$

The Grassmann-Cayley algebra on $V$ is defined as the vector space $\Lambda(V)$ with the operations join and meet. These operations are both associative, distributive over addition, and anticommutative.

\subsection{The superbracket and its decomposition}

Many researchers in the field of parallel robots have explored the determinant of the $6 \times 6$ Jacobian matrix $\mathbf{J}$ to analyze the singularities. The columns of $\mathbf{J}^{T}$ of a PM are usually six Plücker vectors. Each Plücker vector, being of six components, is the support of an extensor of step 1 in the 5-dimensional projective space $\mathbb{P}^{5}$ (a Plücker vector corresponds to a point in $\mathbb{P}^{5}$ ). The join (also called superjoin) of these six 1-extensors in $\mathbb{P}^{5}$ is equal to the determinant of $\mathbf{J}^{T}$, up to scale. This determinant matrix, whose columns are the Plücker coordinates of six lines (2-extensors), is called the superbracket in GCA $\Lambda\left(V^{(2)}\right)[20,21]$. Thus, a singularity occurs when this superbracket vanishes. 
The superbracket is an expression involving 12 points selected on six projective lines (2-extensors) and can be developed into a linear combination of 24 bracket monomials [2, 22], each one being the product of three brackets of four projective points:

$$
[\mathrm{ab}, \mathrm{cd}, \mathrm{ef}, \mathrm{gh}, \mathrm{ij}, \mathrm{kl}]=\sum_{i=1}^{24} y_{i}
$$

where

$$
\begin{aligned}
& y_{1}=-[\mathrm{abcd}][\mathrm{efgi}][\mathrm{hjkl}] \quad y_{2}=[\mathrm{abcd}][\mathrm{efhi}][\mathrm{gjkl}] \quad y_{3}=[\mathrm{abcd}][\mathrm{efgj}][\mathrm{hikl}] \\
& y_{4}=-[\mathrm{abcd}][\mathrm{efhj}][\mathrm{gikl}] \quad y_{5}=[\mathrm{abce}][\mathrm{dfgh}][\mathrm{ijkl}] \quad y_{6}=-[\mathrm{abde}][\mathrm{cfgh}][\mathrm{ijkl}] \\
& y_{7}=-[\mathrm{abcf}][\mathrm{degh}][\mathrm{ijkl}] \quad y_{8}=[\mathrm{abdf}][\mathrm{cegh}][\mathrm{ijkl}] \quad y_{9}=-[\mathrm{abce}][\mathrm{dghi}][\mathrm{fjkl}] \\
& y_{10}=[\mathrm{abde}][\mathrm{cghi}][\mathrm{fjkl}] \quad y_{11}=[\mathrm{abcf}][\mathrm{dghi}][\mathrm{ejkl}] \quad y_{12}=[\mathrm{abce}][\mathrm{dghj}][\mathrm{fikl}] \\
& y_{13}=-[\operatorname{abdf}][\mathrm{cghi}][\mathrm{ejkl}] \quad y_{14}=-[\mathrm{abde}][\mathrm{cghj}][\mathrm{fikl}] \quad y_{15}=-[\mathrm{abcf}][\operatorname{dgh} j][\mathrm{eikl}] \\
& y_{16}=[\mathrm{abdf}][\mathrm{cghj}][\mathrm{eikl}] \quad y_{17}=[\mathrm{abcg}][\mathrm{defi}][\mathrm{hjkl}] \quad y_{18}=-[\mathrm{abdg}][\mathrm{cefi}][\mathrm{hjkl}] \\
& y_{19}=-[\mathrm{abch}][\operatorname{defi}][\mathrm{gjkl}] \quad y_{20}=-[\mathrm{abcg}][\operatorname{def} j]\left[\text { hikl] } \quad y_{21}=\right.\text { [abdh][cefi][gjkl] } \\
& y_{22}=[\mathrm{abdg}][\mathrm{cefj}][\mathrm{hikl}] \quad y_{23}=[\mathrm{abch}][\operatorname{defj}][\mathrm{gikl}] \quad y_{24}=-[\mathrm{abdh}][\mathrm{cef} j][\mathrm{gikl}]
\end{aligned}
$$

A bracket $[a b c d]$ is null if and only if (iff) the projective points $a, b, c$ and $d$ are coplanar. The bracket of four projective points is defined as the determinant of the matrix whose columns are the homogeneous coordinates of these points. It is noteworthy that a bracket containing one finite point and three distinct points at infinity does not depend on the finite point. Indeed, let $g, \underline{i}, \underline{j}$ and $\underline{k}$ be one given finite point and three disctinct points at infinity, respectively. Then,

$$
[\mathrm{g} \underline{\mathrm{i}} \underline{\mathrm{k}} \underline{\mathrm{j}}]=\left|\begin{array}{cccc}
g_{1} & i_{1} & k_{1} & j_{1} \\
g_{2} & i_{2} & k_{2} & j_{2} \\
g_{3} & i_{3} & k_{3} & j_{3} \\
1 & 0 & 0 & 0
\end{array}\right|=\left|\begin{array}{ccc}
i_{1} & k_{1} & j_{1} \\
i_{2} & k_{2} & j_{2} \\
i_{3} & k_{3} & j_{3}
\end{array}\right|=[\underline{i} \underline{\mathrm{k}} \underline{\mathrm{j}}]=[\mathrm{x} \underline{\mathrm{i}} \underline{\mathrm{k}} \underline{\mathrm{j}}]
$$

where $\mathrm{x}$ can be any finite point.

\subsection{Projective space}

The 3-dimensional projective space $\mathbb{P}_{3}$ is characterized by the affine space $\mathbb{R}^{3}$ in addition to the plane at infinity $\Omega_{\infty}$. It is noteworthy that the coordinates of a projective element are determined up to a scalar multiple. A projective point has four homogeneous coordinates whereas a projective line has six Plücker coordinates represented by its Plücker coordinate vector. The following properties highlight the relations between projective elements:

- A finite point, $A$, is represented by its homogeneous coordinates vector a $=\left(a_{1}, a_{2}, a_{3}, 1\right)^{T}$, the first three coordinates being its Cartesian coordinates in $\mathbb{R}^{3}$;

- A finite line, $\mathscr{L}$, is represented by its Plücker coordinates vector $F=(\mathbf{s} ; \mathbf{r} \times \mathbf{s})$; where $\mathbf{s}$ is the unit vector of $\mathscr{L}, \mathbf{r}$ is the position vector of any point on $\mathscr{L}$ and $(\mathbf{r} \times \mathbf{s})$ represents the moment of $\mathscr{L}$ with respect to the origin; 


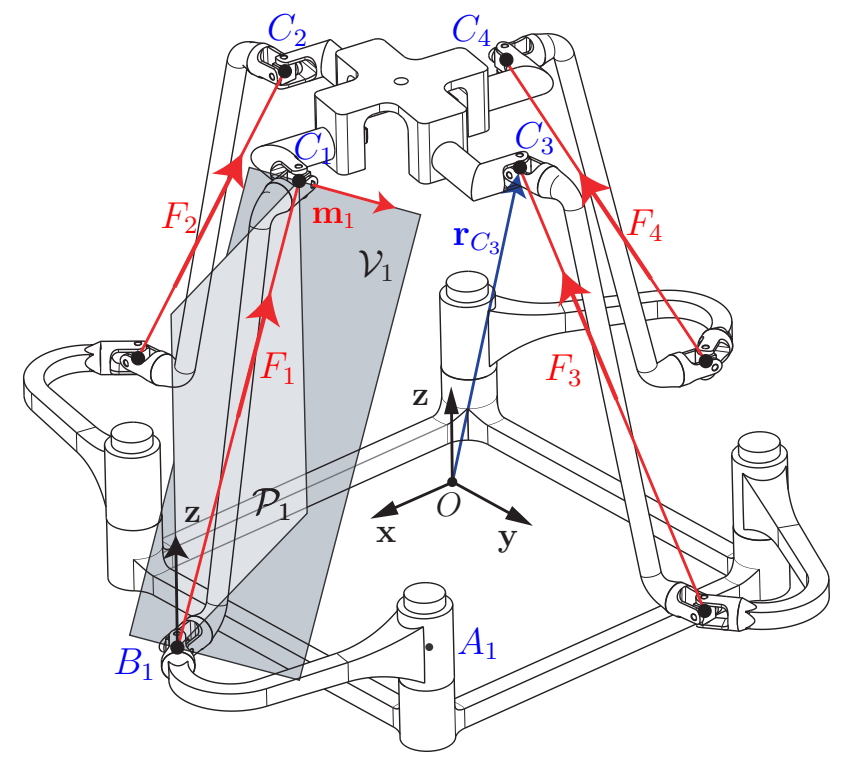

(a) CAD model.

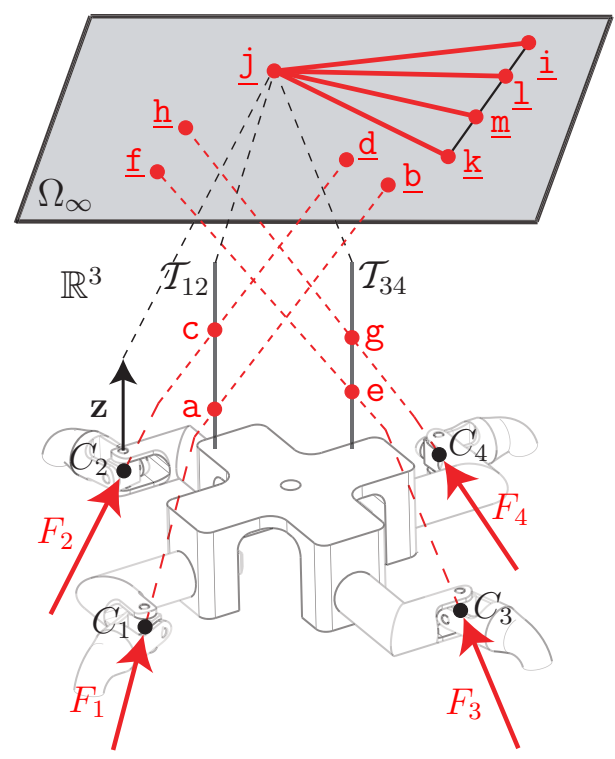

(b) Wrench graph in $\mathbb{P}_{3}$.

Figure 1: A 4-ㅌUU PM.

- Let underlined points denote points at infinity. Any finite line, $F=(\mathbf{s} ; \mathbf{r} \times \mathbf{s})$, has a unique point at infinity $\underline{c}=(\mathbf{s} ; 0)$. This point only depends on the line direction and is determined up to scale. Accordingly, if $\mathrm{a}$ and $\mathrm{b}$ are two finite points on $F$, then $\underline{c}=\mathrm{b}-\mathrm{a}$. Furthermore, all finite lines directed along $\mathbf{s}$ intersect at one common point at infinity, namely, $\underline{\mathbf{c}}$;

- All finite planes of normal vector $\mathbf{m}$, have a common line at infinity. This line is given by: $M=(\mathbf{0} ; \mathbf{m})$ and passes through the point at infinity on any finite line orthogonal to $\mathbf{m}$;

- Two lines at infinity $M_{1}=\left(\mathbf{0} ; \mathbf{m}_{1}\right)$ and $M_{2}=\left(\mathbf{0} ; \mathbf{m}_{2}\right)$ intersect at a unique point at infinity $\underline{\mathrm{g}}=\left(\mathbf{m}_{1} \times \mathbf{m}_{2} ; 0\right)$.

\section{JACOBIAN MATRIX OF THE 4-RUUU PM}

\subsection{Architecture review and kinematic modeling}

Figure 1(a) represents the CAD model of a 4- $\underline{\mathrm{R} U U} \mathrm{PM}$, which consists of a fixed base and a moving platform connected to each other with four identical $\underline{R} U U$ limbs. The input of the mechanism is provided by four revolute actuators attached to the base. From [12], the geometric characteristics of each limb are:

1. Each limb is composed of five revolute joints. The second and the third joints, as well as the fourth and the fifth joints, are built with intersecting and perpendicular axes and are thus assimilated to $\mathrm{U}$-joints centered at points $B_{i}$ and $C_{i}$, respectively;

2. The axes of the third and the fourth revolute joints of the $i$ th limb are parallel to $\mathbf{m}_{i}$. These axes define a plane $\mathscr{V}_{i}$. Let $\mathbf{f}_{i}$ be the unit vector of line $B_{i} C_{i}$. Thus, plane $\mathscr{V}_{i}$ has $\left(\mathbf{m}_{i} \times \mathbf{f}_{i}\right)$ as normal 
vector;

3. The first, the second and the fifth revolute joints of the $i$ th limb have axes parallel to a fixed direction along $\mathbf{z}$. Plane $\mathscr{P}_{i}$, defined by the axes of the second and the last revolute joints, has $\left(\mathbf{z} \times \mathbf{f}_{i}\right)=\mathbf{m}_{i}$ as normal vector. However, plane $\mathscr{P}_{i}$ degenerates into a line if $\mathbf{f}_{i}$ is parallel to $\mathbf{z}$.

\subsection{Constraint analysis}

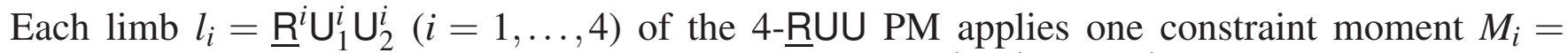
$\left(\mathbf{0} ; \mathbf{m}_{i} \times \mathbf{z}\right)$ reciprocal to the twists associated with joints $\underline{\mathrm{R}}^{i}, \mathrm{U}_{1}^{i}$ and $\mathrm{U}_{2}^{i}$. Vectors $\mathbf{m}_{i} \times \mathbf{z}$ are orthogonal to $\mathbf{z}$. Thus, in a non singular configuration, the four constraint wrenches $M_{i}$ form a 2-system $W_{4 R U U}^{c}$, namely, the constraint wrench system of the PM:

$$
\mathrm{W}_{4 R U U}^{c}=\operatorname{span}\left(M_{i}\right) ; i=1, \ldots, 4
$$

The limbs of the 4-ㅁUU PM can each apply one constraint moment but altogether they limit only two dof, namely, two rotations of the moving platform. Thus, it is an over-constrained SMG. The moving platform cannot rotate about an axis of direction orthogonal to $\mathbf{z}$. It provides three independent translations and one rotation about an axis of fixed direction along $\mathbf{z}$. By locking the actuator of the $i$ th limb, an additional constraint appears, which is called the limb actuation wrench. It is a pure force $F_{i}=\left(\mathbf{f}_{i} ; \mathbf{r}_{C_{i}} \times \mathbf{f}_{i}\right)$ where $\mathbf{f}_{i}$ is the unit vector of $\left(B_{i} C_{i}=\mathscr{P}_{i} \cap \mathscr{V}_{i}\right)$ and $\mathbf{r}_{C_{i}}$ is the position vector of point $C_{i}$. In a non-singular configuration, the actuation wrench system of the PM is a 4-system expressed as:

$$
\mathrm{W}_{4 R U U}^{a}=\operatorname{span}\left(F_{i}\right) ; i=1, \ldots, 4
$$

Based on the constraint analysis, the rows of $\mathbf{J}$ of the 4-RUU PM can be composed of four independent zero pitch wrenches within $W_{4 R U U}^{a}$ plus two independent infinite pitch wrenches within $W_{4 R U U}^{c}$. However, a parallel singularity occurs when the system spanned by the four actuation forces and the four constraint moments becomes a $(n<6)$-system.

\section{LIMB SINGULARITIES}

A limb singularity is similar to the singularity of a serial manipulator. It occurs for the 4-RUU PM when a limb kinematic screw system (twist system) degenerates. Consequently, the platform loses one dof in such a configuration. Let us consider the twist system of the $i$ th limb. It can be expressed in matrix form as follows:

$$
\left[\begin{array}{ccccc}
\mathbf{z} & \mathbf{z} & \mathbf{m}_{i} & \mathbf{m}_{i} & \mathbf{z} \\
\mathbf{z} \times \mathbf{r}_{A_{i}} & \mathbf{z} \times \mathbf{r}_{B_{i}} & \mathbf{m}_{i} \times \mathbf{r}_{B_{i}} & \mathbf{m}_{i} \times \mathbf{r}_{C_{i}} & \mathbf{z} \times \mathbf{r}_{C_{i}}
\end{array}\right]
$$

After some linear transformations, Eq. (11) becomes:

$$
\left[\begin{array}{ccccc}
\mathbf{z} & \mathbf{m}_{i} & \mathbf{0} & \mathbf{0} & \mathbf{0} \\
\mathbf{z} \times \mathbf{r}_{A_{i}} & \mathbf{m}_{i} \times \mathbf{r}_{B_{i}} & \mathbf{z} \times\left(\mathbf{r}_{B_{i}}-\mathbf{r}_{A_{i}}\right) & \mathbf{z} \times\left(\mathbf{r}_{C_{i}}-\mathbf{r}_{A_{i}}\right) & \mathbf{m}_{i} \times \mathbf{f}_{i}
\end{array}\right]
$$

where $\mathbf{f}_{i}=\mathbf{r}_{C_{i}}-\mathbf{r}_{B_{i}}$. Since $\mathbf{z}$ and $\mathbf{m}_{i}$ correspond to two independent directions, this matrix is rank deficient whenever its last three columns (corresponding to infinite-pitch twists) become linearly 


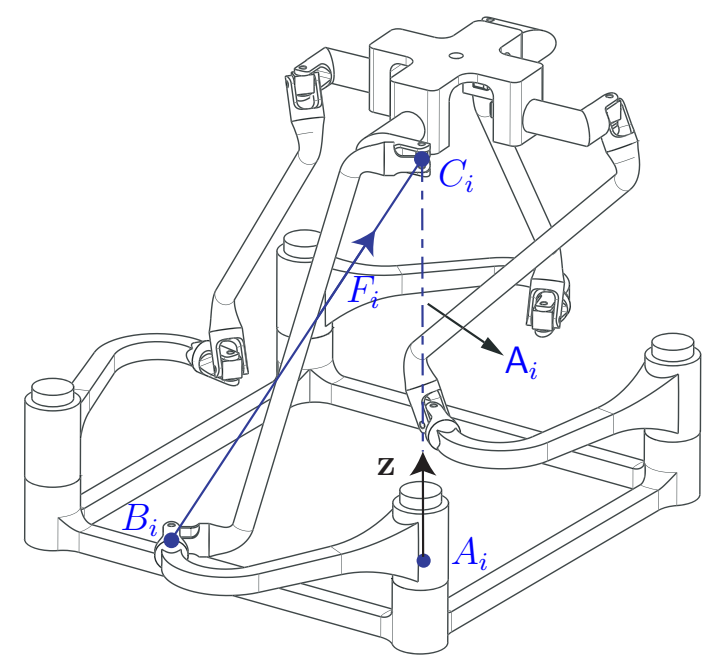

(a) A limb singularity, condition a.1.

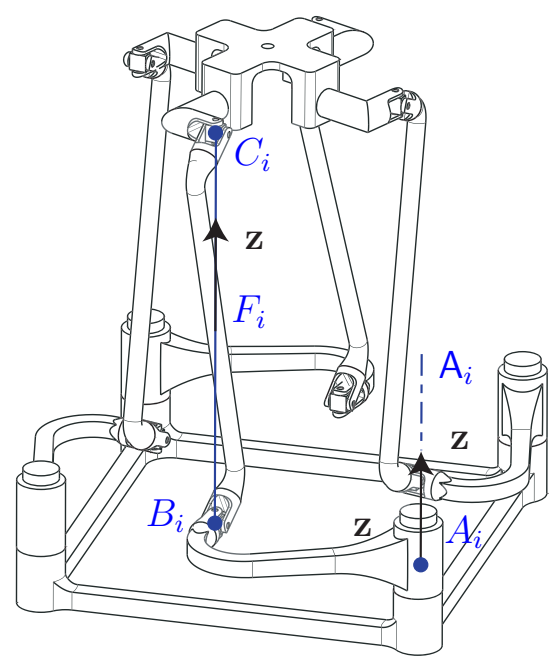

(b) A limb singularity, condition a.2.

Figure 2: Limb singularities.

dependent. On the other hand, in any robot configuration one has: $A_{i}, B_{i}$ and $C_{i}$ are three distinct points, $A_{i} B_{i} \perp \mathbf{z}$ and $\mathbf{m}_{i} \nVdash \mathbf{f}_{i}$. Thus, the $i$ th limb of the 4-RUU PM may exhibit a limb singularity whenever:

a.1 $\left(\mathbf{r}_{C_{i}}-\mathbf{r}_{A_{i}}\right)\left\|\mathbf{z} \Leftrightarrow A_{i} C_{i}\right\| \mathbf{z}$. In that case, $F_{i}=\left(\mathbf{f}_{i} ; \mathbf{r}_{C_{i}} \times \mathbf{f}_{i}\right)$ crosses $\mathrm{A}_{i}$ at point $C_{i}$. As a result, $F_{i}$ acts as a constraint force and the 4-RUU PM loses the translational dof along $\mathbf{f}_{i}$. Figure 2(a) illustrates such a configuration;

a.2 $\left(\mathbf{r}_{C_{i}}-\mathbf{r}_{B_{i}}\right)\left\|\mathbf{z} \Leftrightarrow \mathbf{f}_{i}\right\| \mathbf{z}$ as shown in Fig. 2(b). In that case, $F_{i}$ crosses $A_{i}$ at infinity, i.e., at point $\underline{j}=(\mathbf{z} ; 0)$. Consequently, the 4- $\underline{R} \cup U$ PM loses the translational dof along $\mathbf{z}$.

\section{SINGULARITY ANALYSIS OF THE 4-RUUU PM USING GCA}

In order to formulate a superbracket expression of the 4-ㅌU PM, one must represent its different wrenches in $\mathbb{P}_{3}$ and then select two points on each Plücker line of $\mathbf{J}$. A finite line (pure force) could be represented in the superbracket either by two finite points or by one finite point and its unique point at infinity. In turn, a line at infinity could be represented by two points at infinity. However, the selection of the foregoing points must highlight as much as possible geometric (coincidence, parallelism, orthogonality, intersection and so on) relations between the wrenches in order to obtain a simplified expression of the superbracket.

As shown in Fig. 1(a), each actuation force of the 4-RUU PM can be expressed as $F_{i}=\left(\mathscr{P}_{i} \cap \mathscr{V}_{i}\right)$, $i=1, \ldots, 4$, plane $\mathscr{P}_{i}$ being of normal $\left(\mathbf{z} \times \mathbf{f}_{i}\right)=\mathbf{m}_{i}$ while plane $\mathscr{V}_{i}$ is of normal $\mathbf{m}_{i} \times \mathbf{f}_{i}$. On the other hand, in a general case, two planes $\mathscr{P}_{i}$ and $\mathscr{P}_{j}(i \neq j)$ intersect at a finite line, namely, $\mathscr{T}_{i j}=$ $\left(\mathscr{P}_{i} \cap \mathscr{P}_{j}\right)$. Such a line is orthogonal to both vectors $\mathbf{z} \times \mathbf{f}_{i}$ and $\mathbf{z} \times \mathbf{f}_{j}$ and is thus directed along z. Therefore, for $i \neq j$, one can find a line $\mathscr{T}_{i j}=\left(\mathscr{P}_{i} \cap \mathscr{P}_{j}\right)$ directed along $\mathbf{z}$ and crossing the two 
actuation forces $F_{i}$ and $F_{j}$. In this vein, let a and $\mathrm{c}$ be the intersection points of $\mathscr{T}_{12}$ with $F_{1}$ and $F_{2}$, respectively. Likewise, let e and $g$ be the intersection points of $\mathscr{T}_{34}$ with $F_{3}$ and $F_{4}$, respectively.

On the other hand, let $\underline{\mathrm{b}}=\left(\mathbf{f}_{1} ; 0\right), \underline{\mathrm{d}}=\left(\mathbf{f}_{2} ; 0\right), \underline{\mathbf{f}}=\left(\mathbf{f}_{3} ; 0\right)$ and $\underline{\mathrm{h}}=\left(\mathbf{f}_{4} ; 0\right)$. Accordingly, the four actuation forces can be expressed as:

$$
F_{1}=\mathrm{ab} \quad ; \quad F_{2}=\underline{\mathrm{d}} \underline{;} \quad ; \quad F_{3}=\mathrm{e} \underline{\mathrm{f}} \quad ; \quad F_{4}=\mathrm{g} \underline{\mathrm{h}}
$$

Now let $\underline{\mathrm{x}}=(\mathbf{x} ; 0)$ and $\underline{\mathrm{y}}=(\mathbf{y} ; 0)$. Hence, line $\underline{\mathrm{x}} \underline{\mathrm{y}}$ collects all points at infinity corresponding to directions orthogonal to $\mathbf{z}$. Let $\underline{j}=(\mathbf{z} ; 0), \underline{\underline{i}}=\left(\mathbf{m}_{1} ; 0\right), \underline{\underline{k}}=\left(\mathbf{m}_{2} ; 0\right), \underline{\underline{I}}=\left(\mathbf{m}_{3} ; 0\right)$ and $\underline{\underline{m}}=\left(\mathbf{m}_{4} ; 0\right)$. Accordingly, the four constraint moments are expressed as:

$$
M_{1}=\underline{\mathrm{i}} \underline{\mathrm{j}} \quad ; \quad M_{2}=\underline{\mathrm{k}} \underline{\mathrm{j}} ; M_{3}=\underline{\mathrm{l}} \underline{\mathrm{j}} ; M_{4}=\underline{\mathrm{m}} \underline{\mathrm{j}}
$$

where $\underline{\underline{i}}, \underline{\mathrm{k}}, \underline{\underline{1}}$ and $\underline{\mathrm{m}}$ belong to $\underline{\mathrm{x}} \underline{\mathrm{y}}$. A wrench graph, representing the projective lines associated with the wrenches of the $4-\underline{R} \cup U$ PM in $\mathbb{P}_{3}$, is given in Fig. 1(b).

\subsection{Superbracket decomposition}

Due to the redundancy of constraints, a superbracket of the 4-RUU PM can be composed of the four actuation forces $F_{i}(i=1, \ldots, 4)$ in addition to two among the four constraint moments expressed in Eq. (13). Thus, one can write $\left(\begin{array}{l}4 \\ 2\end{array}\right)=\mathrm{C}_{4}^{2}=6$ superbrackets $S_{j}(j=1, \ldots, 6)$. However, a parallel singularity occurs when the six possible superbrackets vanish simultaneously. For example, the superbracket $S_{1}$ involving the two constraint moments $\underline{\underline{i} j}$ and $\underline{\mathrm{k}} \underline{\mathrm{j}}$ takes the form:

$$
S_{1}=[a \underline{\mathrm{b}}, \mathrm{e} \underline{\mathrm{f}}, \mathrm{c} \underline{\mathrm{d}}, \mathrm{g} \underline{\mathrm{h}}, \underline{\mathrm{i}} \underline{\mathrm{j}}, \underline{\mathrm{k}} \underline{\mathrm{j}}]
$$

From Eq. (7), $S_{1}$ can be decomposed into a linear combination of 24 bracket monomials, which leads to only five non-zero monomials as follows:

$$
\begin{aligned}
& S_{1}=-[a \underline{b} e \underline{f}][c \underline{d} \underline{h} \underline{j}][g \underline{i} \underline{k} \underline{j}]-[a \underline{b} e \underline{d}][\underline{f} g \underline{h} \underline{j}][c \underline{i} \underline{k} \underline{j}]+[a \underline{b} e \underline{h}][\underline{f} c \underline{d} \underline{j}][g \underline{i} \underline{k} \underline{j}] \\
& \text { - }[a \underline{b} \underline{f} \underline{h}][\text { ec } \underline{d} \underline{j}][g \underline{i} \underline{k} \underline{j}]+[a \underline{b} \underline{f} \underline{d}][\operatorname{eg} \underline{h} \underline{j}][c \underline{i} \underline{k} \underline{j}]
\end{aligned}
$$

From Eq. (8), one has $[\mathrm{g} \underline{\underline{i}} \underline{\mathrm{k}} \underline{j}]=[\underline{\underline{i}} \underline{\mathrm{j}} \underline{j}]$. Furthermore, since points e, $\mathrm{g}$ and $\underline{j}$ belong to the same projective line, namely, to $\mathscr{T}_{34}$, the bracket $[\operatorname{eg} \underline{\mathrm{h}} \underline{j}]$ is null and therefore: [a $\left.\underline{b} \underline{\underline{f}} \underline{\mathrm{d}}\right][\operatorname{eg} \underline{\mathrm{h}} \underline{j}][\mathrm{c} \underline{\mathrm{i}} \underline{\mathrm{k}} \underline{j}]=0$. Thus, Eq. (15) becomes:

$$
S_{1}=[\underline{i} \underline{k} \underline{j}](-[a \underline{b} e \underline{f}][c \underline{d} \underline{h} \underline{j}]-[a \underline{b} \text { e } \underline{d}][\underline{f} g \underline{h} \underline{j}]+[a \underline{b} e \underline{h}][\underline{f} \mathrm{c} \underline{d} \underline{j}]-[a \underline{b} \underline{f} \underline{h}][e c \underline{d} \underline{j}])
$$

From the syzygies or Grassmann-Plücker relations $[2,20]$, it follows that:

$$
[a \underline{b} \text { e c }][\underline{f} \underline{d} \underline{h} \underline{j}]=+[a \underline{b} \text { e } \underline{f}][c \underline{d} \underline{h} \underline{j}]+[a \underline{b} \text { e } \underline{d}][\underline{f} c \underline{h} \underline{j}]+[a \underline{b} \text { e } \underline{h}][\underline{f} \underline{d} \mathrm{c} c \underline{j}]+[a \underline{b} \text { e } \underline{j}][\underline{f} \underline{d} \underline{h} \mathrm{c}]
$$

On the other hand, $[\underline{f} \underline{\mathrm{d}} \underline{\mathrm{h}} \underline{j}]=0$ and $[\underline{\mathrm{f}} \underline{\mathrm{d}} \mathrm{j} \underline{j}]=-[\underline{\mathrm{f}} \mathrm{c} \underline{\mathrm{d}} \underline{j}]$. From Eq. (8) $[\underline{\mathrm{f}} g \underline{\mathrm{h}} \underline{j}]=[\underline{f} \mathrm{c} \underline{\mathrm{h}} \underline{j}]$. Therefore,

$$
[a \underline{b} e \underline{j}][\underline{f} \underline{d} \underline{h} c]=-[a \underline{b} e \underline{f}][c \underline{d} \underline{h} \underline{j}]-[a \underline{b} e \underline{d}][\underline{f} c \underline{h} \underline{j}]+[a \underline{b} e \underline{h}][\underline{f} c \underline{d} \underline{j}]
$$




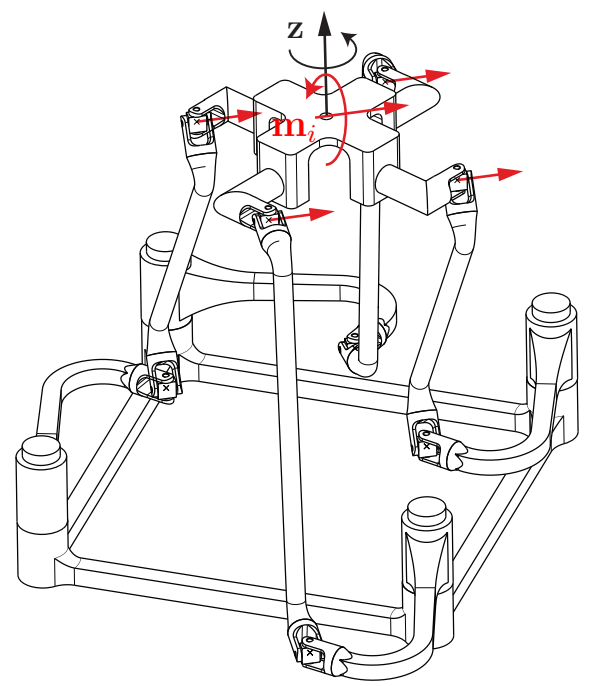

(a) A constraint singular configuration.

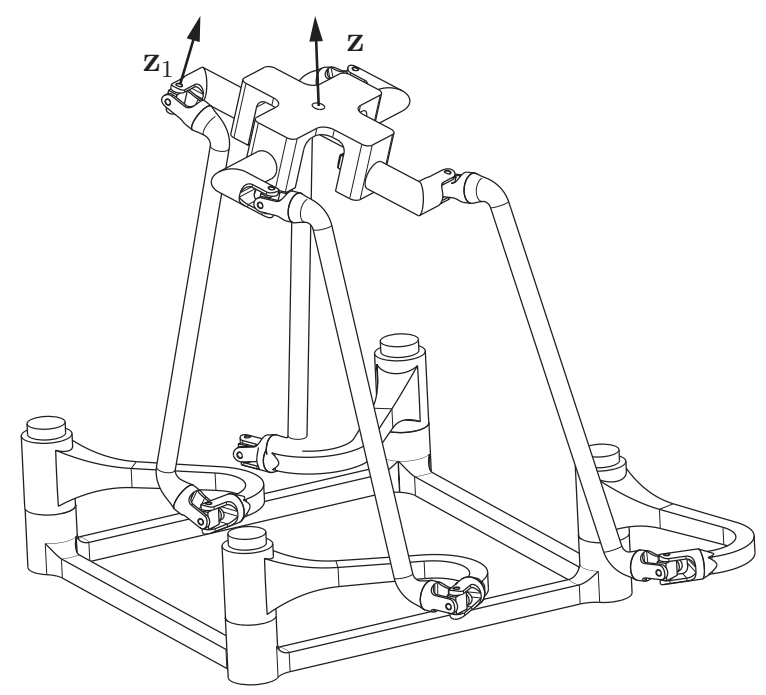

(b) A coupled motion.

Figure 3: Two critical configurations of the 4-ㅁUU PM.

As a result, Eq. (16) becomes:

$$
S=\underbrace{[\underline{i} \underline{\underline{k}} \underline{j}]}_{A_{1}} \underbrace{([\mathrm{a} \underline{\mathrm{b}} \mathrm{e} \underline{\mathrm{j}}][\underline{\mathrm{f}} \underline{\mathrm{d}} \underline{\mathrm{h}} \mathrm{c}]-[\mathrm{a} \underline{\mathrm{b}} \underline{\mathrm{f}} \underline{\mathrm{h}}][\mathrm{e} \mathrm{c} \underline{\mathrm{d}} \underline{\mathrm{j}}])}_{B}
$$

Term $A_{1}=[\mathrm{g} \underline{\underline{i}} \underline{\mathrm{k}} \underline{j}]$ in Eq. (19) depends only on the chosen constraint moments $\underline{i} \underline{j}$ and $\underline{\mathrm{k}} \underline{j}$ whereas term $B$ does not depend on the choice of points $\underline{\underline{i}}$ and $\underline{\mathrm{k}}$. Consequently, term $B$ is a common factor of the six possible superbrackets:

$$
S_{j}=A_{j} B \quad ; \quad j=1, \ldots, 6
$$

where $A_{1}=[\underline{\underline{i}} \underline{\mathrm{j}} \underline{\mathrm{j}}], A_{2}=[\underline{\mathrm{i}} \underline{\mathrm{l}} \underline{\mathrm{j}}], A_{3}=[\underline{\mathrm{i}} \underline{\mathrm{m}} \underline{\mathrm{j}}], A_{4}=[\underline{\mathrm{k}} \underline{\mathrm{l}} \underline{\mathrm{j}}], A_{5}=[\underline{\mathrm{k}} \underline{\mathrm{m}} \underline{\mathrm{j}}]$ and $A_{6}=[\underline{\mathrm{l}} \underline{\mathrm{j}} \underline{\mathrm{j}}]$.

\subsection{Condition for constraint singularities}

Constraint singularities correspond to the degeneracy of the constraint wrench system of the manipulator. In such configurations, the manipulator loses some constraints and, as a result, its moving platform gains one or several dof. Accordingly, a constraint singularity of the 4- $\underline{R U U}$ PM occurs when the four constraint moments $M_{i}(i=1, \ldots, 4)$ form a $n<2$-system, i.e., when all terms $A_{j}$ $(j=1, \ldots, 6)$ expressed in Eq. (20) vanish simultaneously. Let us consider bracket $[\mathrm{g} \underline{i} \underline{\mathrm{k}} \underline{j}]$, namely, term $A_{1}$. This bracket vanishes iff points $\underline{\underline{i}}, \underline{\mathrm{k}}$ and $\underline{j}$ belong to the same projective line. Since point $\underline{j}$ corresponds to the fixed direction of vector $\mathbf{z}$, it is a fixed point. Points $\underline{\underline{i}}$ and $\underline{k}$ correspond to two directions orthogonal to $\mathbf{z}$ and, therefore, these points belong to a line that cannot pass through point $\underline{j}$ unless $\underline{\underline{i}}$ and $\underline{\mathrm{k}}$ are coincident. Consequently, all terms $A_{j}$ vanish simultaneously iff points $\underline{\underline{i}}, \underline{\mathrm{k}}, \underline{\underline{l}}$, and $\underline{m}$ become all coincident. As a result, the 4- $\underline{R} U U$ PM reaches a constraint singularity iff:

$$
\mathbf{m}_{1}\left\|\mathbf{m}_{2}\right\| \mathbf{m}_{3} \| \mathbf{m}_{4}
$$


In such a configuration, the constraint wrench system of the manipulator degenerates into a 1-system and the moving platform gains one extra dof, namely, the rotation about an axis directed along the common direction of $\mathbf{m}_{i}(i=1, \ldots, 4)$, as shown in Fig. 3(a). In such a critical configuration, if the moving platform rotates about an axis directed along $\mathbf{z}$, then the robot will come back to a nonsingular configuration.

On the other hand, in a constraint singular configuration, if the moving platform rotates about an axis directed along $\mathbf{m}_{i}$, the revolute joints attached to the moving platform will no longer be directed along $\mathbf{z}$. As a consequence, the constraint wrench of each limb becomes a wrench of finite pitch (a combination of a force and a moment). In that case, the moving platform has neither pure constraint moments nor pure constraint forces. Moreover, the limbs constrain neither a pure rotation nor a pure translation. Such a configuration is shown in Fig. 3(b) and corresponds to a coupled motion.

\subsection{Conditions for actuation singularities}

In this paper, the actuation singularities correspond to configurations in which $\mathbf{J}$ is rank deficient while the constraint wrench system does not degenerate. In such configurations, the motion of the moving platform becomes uncontrollable, namely, the actuators cannot control the motion of the moving platform. According to Eq. (20), these singularities are related to the vanishing conditions of term $B$. In order to obtain geometric and vector conditions for actuation singularities, term $B$ is expressed in a more compact form by considering the following bracket simplifications:

- $[\underline{f} \underline{\mathrm{d}} \underline{\mathrm{h}} \mathrm{c}]=[\mathrm{c} \underline{\mathrm{d}} \underline{\mathrm{f}} \underline{\mathrm{h}}]=[\mathrm{a} \underline{\mathrm{d}} \underline{\mathrm{f}} \underline{\mathrm{h}}]$;

- Since $\underline{j}=c-a,[a \underline{b} e \underline{j}]=[(c-\underline{j}) \underline{b} e \underline{j}]=[c \underline{b}$ e $\underline{j}]=[e c \underline{b} \underline{j}]$.

Accordingly,

$$
B=[\mathrm{a} \underline{\mathrm{d}} \underline{\mathrm{f}} \underline{\mathrm{h}}][\mathrm{e} \mathrm{c} \underline{\mathrm{b}} \underline{\mathrm{j}}]-[\mathrm{a} \underline{\mathrm{b}} \underline{\mathrm{f}} \underline{\mathrm{h}}][\mathrm{e} \mathrm{c} \underline{\mathrm{d}} \underline{\mathrm{j}}]=[\mathrm{a} \underline{\mathrm{d}} \underline{\mathrm{f}} \underline{\mathrm{h}}][\mathrm{e} \mathrm{c} \underline{\mathrm{b}} \underline{\mathrm{j}}]=(\mathrm{a} \underline{\mathrm{f}} \underline{\mathrm{h}}) \wedge(\mathrm{e} \mathrm{c} \underline{\mathrm{j}}) \wedge(\underline{\mathrm{d}} \underline{\mathrm{b}})
$$

where the dotted letters stand for the permuted elements as explained in [16, 20]. From Eq. (22), term $B$ is the meet of three geometric entities, namely,

1. $(\mathrm{a} \underline{\underline{f}} \underline{\mathrm{h}})$ is a finite plane having $\mathbf{f}_{3} \times \mathbf{f}_{4}$ as normal vector;

2. $(e \subset j)$ is the finite plane containing the finite points e and $c$ and the unit vector $\mathbf{z}$. Since plane (ecji ) contains lines $\mathscr{T}_{12}$ and $\mathscr{T}_{34}$ (Fig. 1(b)), the line at infinity of plane $(\mathrm{ec} \underline{j})=$ $\operatorname{span}\left(\mathscr{T}_{12}, \mathscr{T}_{34}\right)$ can be expressed as $(\underline{u} j)$ where $\underline{u}=(\mathbf{u} ; 0)$ and $\mathbf{u}$ is the unit vector of a finite line non-parallel to $\mathbf{z}$ and lying in plane $(\mathrm{ec} \underline{j})$, i.e., crossing $\mathscr{T}_{12}$ and $\mathscr{T}_{34}$. Accordingly, plane $(\mathrm{e} \subset \underline{\mathrm{j}})$ has $\mathbf{u} \times \mathbf{z}$ as normal vector. It should be noted that $\underline{u}$ and $\mathbf{u}$ exist unless $\mathscr{T}_{12} \equiv \mathscr{T}_{34}$;

3. $(\underline{\mathrm{d}} \underline{\mathrm{b}})$ is the line at infinity of all parallel finite planes containing the unit vectors $\mathbf{f}_{1}$ and $\mathbf{f}_{2}$, i.e., having $\mathbf{f}_{1} \times \mathbf{f}_{2}$ as normal vector. 


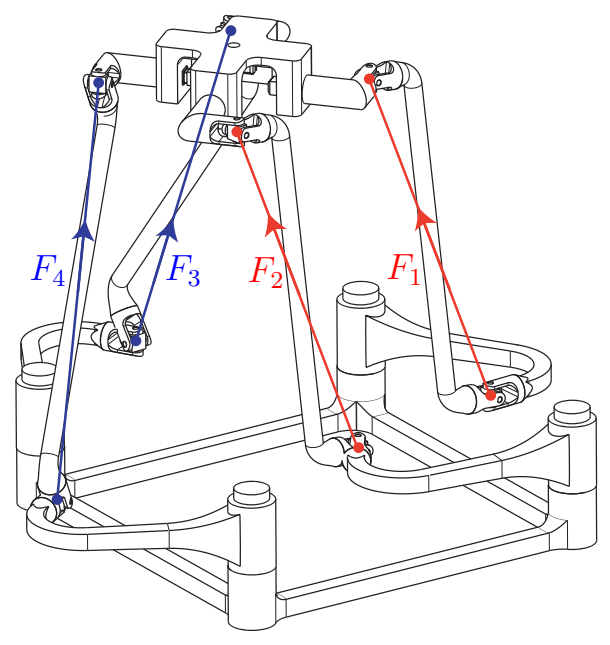

(a) $F_{1}$ and $F_{2}$ are parallel.

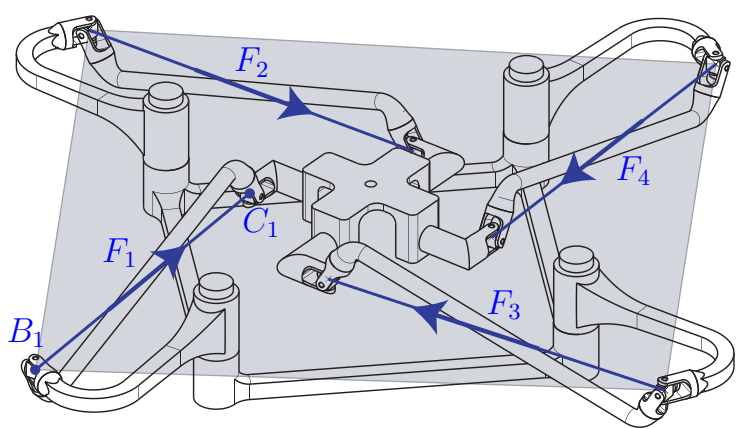

(b) All actuation forces are coplanar.

Figure 4: Two actuation singular configurations of the 4-ㅁUU PM.

An actuation singularity occurs iff term $B$ of Eq. (22) vanishes. It amounts to the following vector form:

$$
\left(\left(\mathbf{f}_{3} \times \mathbf{f}_{4}\right) \times(\mathbf{u} \times \mathbf{z})\right) \cdot\left(\mathbf{f}_{2} \times \mathbf{f}_{1}\right)=0
$$

From Eqs. (22) and (23), an actuation singularity of the 4-RUU PM occurs whenever:

(a) Plane (a $\underline{\underline{f}} \underline{\mathrm{h}})$ degenerates, which happens iff $\underline{\mathrm{f}} \equiv \underline{\mathrm{h}} \Leftrightarrow \mathbf{f}_{3} \| \mathbf{f}_{4}$, i.e., $F_{3}$ and $F_{4}$ are parallel;

(b) Plane (e c j $)$ degenerates, which happens iff points e, $\mathrm{c}$ and $\underline{\mathrm{j}}$ are aligned. In that case, $\mathscr{T}_{12} \equiv \mathscr{T}_{34}$ and $\mathbf{u}=\overline{\mathbf{0}}$;

(c) Line ( $\underline{\mathrm{d}} \underline{\mathrm{b}})$ degenerates, which happens iff $\underline{\mathrm{d}} \equiv \underline{\mathrm{b}} \Leftrightarrow \mathbf{f}_{2} \| \mathbf{f}_{1}$ as shown in Fig. 4(a);

(d) Plane (a $\underline{f} \underline{h})$ coincides with plane (e c $\underline{j})$. Since point a lies in plane $(\mathrm{ec} \underline{j})$, the condition $(\mathrm{a} \underline{\mathrm{f}} \underline{\mathrm{h}}) \equiv$ $(\operatorname{ec} \underline{j})$ amounts to $(\underline{\mathrm{f}} \underline{\mathrm{h}}) \equiv(\underline{\mathrm{u}} \underline{\mathrm{j}})$. In that case, $\left(\mathbf{f}_{3} \times \mathbf{f}_{4}\right) \|(\mathbf{u} \times \mathbf{z})$, i.e., $\mathbf{f}_{3}, \overline{\mathbf{f}}_{4}, \mathbf{u}$ and $\mathbf{z}$ are orthogonal to a given direction;

(e) Line $(\underline{d} \underline{b})$ lies in plane $(a \underline{f} \underline{h})$. In such a case, $\left(\mathbf{f}_{2} \times \mathbf{f}_{1}\right) \|\left(\mathbf{f}_{3} \times \mathbf{f}_{4}\right)$. For example, if the four actuation forces are coplanar, as shown in Fig. 4(b);

(f) Line ( $\underline{\mathrm{d}} \underline{\mathrm{b}})$ lies in plane $(\mathrm{e} \mathrm{c} \underline{\mathrm{j}})$. In such a case, $\left(\mathbf{f}_{2} \times \mathbf{f}_{1}\right) \|(\mathbf{u} \times \mathbf{z})$;

(g) The intersection line of planes $(a \underline{f} \underline{h})$ and $(e \subset \underline{j})$ coincides with line $(\underline{d} \underline{b})$. Since planes $(a \underline{f} \underline{h})$ and $(\mathrm{e} \subset \underline{j})$ contain point $a$, they intersect at a line at infinity iff they coincide. Accordingly, condition $(\mathbf{g})$ amounts to $(\underline{\underline{f}} \underline{\mathrm{h}}) \equiv(\underline{\mathrm{u}} \underline{\mathrm{j}}) \equiv(\underline{\mathrm{d}} \underline{\mathrm{b}})$, i.e., $\left(\mathbf{f}_{3} \times \mathbf{f}_{4}\right)\|(\mathbf{u} \times \mathbf{z})\|\left(\mathbf{f}_{2} \times \mathbf{f}_{1}\right)$; 
Table 1: Actuation singularity conditions of the 4- $\underline{\mathrm{RUU}} \mathrm{PM}$.

\begin{tabular}{l|l|l}
\hline \hline Case & \multicolumn{1}{|c|}{ Vector form } & \multicolumn{1}{c}{ Algebraic form } \\
\hline (a) & $\mathbf{f}_{3} \| \mathbf{f}_{4}$ & $\underline{\underline{f}} \equiv \underline{\mathrm{h}}$ \\
\hline (b) & $\mathbf{u}=\mathbf{0}$ & $\mathrm{e}, \mathrm{c}$ and $\underline{\mathrm{j}}$ are aligned \\
\hline (c) & $\mathbf{f}_{2} \| \mathbf{f}_{1}$ & $\underline{\mathrm{d}} \equiv \underline{\mathrm{b}}$ \\
\hline (d) & $\left(\mathbf{f}_{3} \times \mathbf{f}_{4}\right) \|(\mathbf{u} \times \mathbf{z})$ & $\underline{\underline{\mathrm{h}}} \equiv \underline{\mathrm{u}} \underline{\mathrm{j}}$ \\
\hline (e) & $\left(\mathbf{f}_{2} \times \mathbf{f}_{1}\right) \|(\mathbf{u} \times \mathbf{z})$ & $\underline{\mathrm{d}} \underline{\mathrm{b}} \equiv \underline{\mathrm{u}} \underline{\mathrm{j}}$ \\
\hline (f) & $\left(\mathbf{f}_{3} \times \mathbf{f}_{4}\right) \|\left(\mathbf{f}_{2} \times \mathbf{f}_{1}\right)$ & $\underline{\underline{\mathrm{h}}} \equiv \underline{\mathrm{d}} \underline{\mathrm{b}}$ \\
\hline (g) & $\left(\mathbf{f}_{3} \times \mathbf{f}_{4}\right)\|(\mathbf{u} \times \mathbf{z})\|\left(\mathbf{f}_{2} \times \mathbf{f}_{1}\right)$ & $\underline{\underline{\mathrm{h}}} \underline{\mathrm{u}} \underline{\mathrm{j}} \equiv \underline{\mathrm{d}} \underline{\mathrm{b}}$ \\
\hline (h) & $\left(\left(\mathbf{f}_{3} \times \mathbf{f}_{4}\right) \times(\mathbf{u} \times \mathbf{z})\right) \perp\left(\mathbf{f}_{2} \times \mathbf{f}_{1}\right)$ & $(\underline{\underline{\mathrm{f}}} \underline{\mathrm{h}} \wedge \underline{\mathrm{u}} \underline{\mathrm{j}}) \in \underline{\mathrm{d}} \underline{\mathrm{b}}$ \\
\hline \hline
\end{tabular}

(h) Let us consider the general case of Eq. (23), namely, the intersection line of planes (a $\underline{f} \underline{h}$ ) and $(e c j)$ crosses line $(\underline{d} \underline{b})$. If planes $(a \underline{f} \underline{h})$ and $(e \subset \underline{j})$ are not coincident (condition $(\mathbf{d}))$, then they will intersect at a finite line $\mathscr{D}$ directed along $\mathbf{n}=\left(\mathbf{f}_{3} \times \mathbf{f}_{4}\right) \times(\mathbf{u} \times \mathbf{z})$. Thus, the point at infinity, $\underline{\mathrm{n}}=(\mathbf{n} ; 0)$, of line $\mathscr{D}$ is the intersection point of lines $(\underline{f} \underline{\mathrm{h}})$ and $(\underline{\mathrm{u}} \underline{\mathrm{j}})$. The finite line $\mathscr{D}$ crosses line ( $\underline{\mathrm{d}} \underline{\mathrm{b}})$ iff $\underline{\mathrm{n}} \in(\underline{\mathrm{d}} \underline{\mathrm{b}})$. In that case, the lines at infinity ( $\underline{\mathrm{f}} \underline{\mathrm{h}})$, ( $\underline{\mathrm{d}} \underline{\mathrm{b}})$ and $(\underline{\mathrm{u}} \underline{\mathrm{j}})$ intersect at

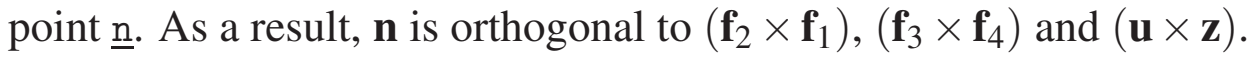

All possible cases of Eq. (23) are expanded in Table 1. It should be noted that Eq. (23) is obtained by considering two lines $\mathscr{T}_{12}$ (crossing $F_{1}$ and $F_{2}$ ) and $\mathscr{T}_{34}$ (crossing $F_{3}$ and $F_{4}$ ). Vector $\mathbf{u}$ in Eq. (23) can be written as $\mathbf{u}_{12}^{34}$, i.e., the unit vector of a finite line non-parallel to $\mathbf{z}$ crossing lines $\mathscr{T}_{12}$ and $\mathscr{T}_{34}$. Accordingly, since a line $\mathscr{T}_{i j}$ exists between each pair of forces $F_{i}$ and $F_{j}$, the vector form of actuation singularities can be generalized as follows:

$$
\left(\left(\mathbf{f}_{i} \times \mathbf{f}_{j}\right) \times\left(\mathbf{f}_{k} \times \mathbf{f}_{l}\right)\right) \cdot\left(\mathbf{u}_{i j}^{k l} \times \mathbf{z}\right)=0
$$

where $(i, j, k, l), i \neq j \neq k \neq l$, is a permutation of $(1,2,3,4)$ and $\mathbf{u}_{i j}^{k l}$ is the unit vector of a finite line non-parallel to $\mathbf{z}$ crossing $\mathscr{T}_{i j}$ and $\mathscr{T}_{k l}$.

\section{CONCLUSIONS}

In this paper, the singularity conditions of 3T1R Parallel Manipulators (PMs) with identical limb structures were investigated through the singularity analysis of the 4-RUU PM based on GrassmannCayley Algebra (GCA). First, the Jacobian matrix $\mathbf{J}$ of the PM was derived using screw theory. Then, a wrench graph that represents the wrenches of the 4-RUU PM, namely, the rows of $\mathbf{J}$ in the 3-dimensional projective space, was obtained. Accordingly, a superbracket was formulated and explored to provide a compact vector expression for the singularity locus, which is difficult to assess using classical linear algebra tools. Finally, all the geometric singularity conditions of the 4-RUU PM were enumerated and some singular configurations were illustrated. 


\section{ACKNOWLEDGEMENTS}

The authors would like to acknowledge the French "Agence Nationale de la Recherche" (Project "SiRoPa", SIngularités des RObots PArallèles), the financial support of the Natural Sciences and Engineering Research Council of Canada (NSERC) and the Canada Research Chair program.

Finally, the authors would like to thank Pierre-Luc Richard and Thierry Laliberte for the CAD models of the 4-RUU parallel manipulator.

\section{REFERENCES}

1. Merlet, J.P. "Singular Configurations of Parallel Manipulators and Grassmann Geometry." The International Journal of Robotics Research, Vol. 8(5), pp. 45-56, 1989.

2. Ben-Horin, P. and Shoham, M. "Singularity Analysis of a Class of Parallel Robots Based on GrassmannCayley Algebra." Mechanism and Machine Theory, Vol. 41(8), pp. 958-970, 2006.

3. Gosselin, C. and Angeles, J. "Singularity Analysis of Closed-Loop Kinematic Chains." IEEE Transactions on Robotics and Automation, Vol. 6(3), pp. 281-290, 1990.

4. Fang, Y. and Tsai, L.W. "Structure Synthesis of a Class of 4-DoF and 5-DoF Parallel Manipulators with Identical Limb Structures." The International Journal of Robotics Research, Vol. 21(9), pp. 799-810, 2002.

5. Conconi, M. and Carricato, M. "A New Assessment of Singularities of Parallel Kinematic Chains." In "Advances in Robot Kinematics: Analysis and Design," pp. 3-12, 2008.

6. Zlatanov, D., Fenton, R.G. and Benhabib, B. "Singularity Analysis of Mechanisms and Robots Via a Velocity-Equation Model of the Instantaneous Kinematics." In "IEEE International Conference on Robotics and Automation," pp. 986-991. San Diego, CA, 1994.

7. Joshi, S.A. and Tsai, L.W. "Jacobian Analysis of Limited-DOF Parallel Manipulators." ASME Journal of Mechanical Design, Vol. 124(2), pp. 254-258, June 2002.

8. Zlatanov, D., Bonev, I. and Gosselin, C.M. "Constraint Singularities of Parallel Mechanisms." In "IEEE International Conference on Robotics and Automation," pp. 496-502. Washington, D.C., May 11-15 2002.

9. Ball., R.S. A Treatise On the Theory of Screws. Cambridge University Press, Cambridge, CA, 1900.

10. Waldron, K.J. The Mobility of Linkages. PhD Thesis, Stanford University, Cambridge, CA, 1969.

11. Hunt, K.H. Kinematic Geometry of Mechanisms. Clarendon Press, Oxford, 1978.

12. Kong, X. and Gosselin, C. Type Synthesis of Parallel Mechanisms, Vol. 33. Springer, Heidelberg, 2007.

13. Merlet, J.P. Parallel Robots, Vol. 128 of Solid Mechanics and Its Applications. Second edition ed.. Springer, 2006.

14. Mbarek, T., Lonij, G. and Corves, B. "Singularity Analysis of a Fully Parallel Manipulator with FiveDegrees-of-Freedom Based on Grassmann Line Geometry." In "12th IFToMM World Congress," Besancon, France, June 18-21 2007.

15. Masouleh, M.T. and Gosselin, C. "Singularity Analysis of 5-RPRRR Parallel Mechanisms via Grassmann Line Geometry." In "ASME 2009 Design Engineering Technical Conferences," No. 86261 in DETC2009. San Diego, California, USA, August 30-September 2009.

16. Ben-Horin, P. and Shoham, M. "Application of Grassmann-Cayley Algebra to Geometrical Interpretation of Parallel Robot Singularities." The International Journal of Robotics Research, Vol. 28(1), pp. 127-141, 2009.

17. Kanaan, D., Wenger, P., Caro, S. and Chablat, D. "Singularity Analysis of Lower-Mobility Parallel 
Manipulators using Grassmann-Cayley Algebra.” IEEE Transactions on Robotics, Vol. 25, pp. 9951004, 2009.

18. Amine, S., Kanaan, D., Caro, S. and Wenger, P. "Constraint and Singularity Analysis of Lower-Mobility Parallel Manipulators with Parallelogram Joints." In "ASME 2010 International Design Engineering Technical Conferences,” No. 28483 in DETC2010. Montreal, Quebec, Canada, August 15-18 2010.

19. Caro, S., Khan, W.A., Pasini, D. and Angeles, J. "The Rule-based Conceptual Design of the Architecture of Serial Schönflies-motion Generators." Mechanism and Machine Theory, Vol. 45(2), pp. 251-260, 2010 .

20. White, N.L. Grassmann-Cayley Algebra and Robotics Applications, Vol. VIII. Handbook of Geometric Computing, 2005.

21. White, N.L. "The Bracket of 2-Extensors.” Congressus Numerantium, Vol. 40, pp. 419-428, 1983.

22. McMillan, T. Invariants of Antisymmetric Tensors. PhD Thesis, University of Florida, 1990. 